\title{
Health stories that mattered in 2019
}

\author{
Cite as: CMAJ 2020 January 20;192:E77-8. doi: 10.1503/cmaj.1095844
}

Posted on cmajnews.com on December 23, 2019

anadian doctors refused to "stay in their lane" in 2019, advocating for national action on gun control, climate change and pharmacare in the lead up to the federal election. But within the profession, entrenched discrimination and poor working conditions remained major challenges. And looming shortages of physicians raised pressing questions about the future of health care in Canada.

\section{Health on the ballot}

The 2019 federal election put health care back on the national agenda with most parties promising to implement some form of national universal pharmacare. In the lead up to the election, pharmacare, gun control and the health impacts of climate change dominated debate.

Early in the year, Canadian physicians joined their American colleagues in declaring that the medical profession has a duty to address firearms safety, drawing fierce opposition from gun lobby groups. One group had supporters file regulatory complaints against a Toronto surgeon, alleging her advocacy on the issue was unprofessional.

The Canadian Paediatric Society warned that firearms injuries are a public health emergency and urged all federal parties to support tougher gun controls. They also called for action on pharmacare but faced criticism for leaving climate change off their advocacy agenda.

Canada's health system ranked among the least green, according to a Lancet Countdown policy brief. One study found that health care in Canada accounted for $4.6 \%$ of national greenhouse gas emissions, contributing to an estimated 23000 years of life lost each year due to disability or early death.
Yet, despite warnings that climate changes poses a major threat to human health, many doctors lacked awareness and confidence that they can make a difference.

\section{Working conditions}

Young doctors demanded change in many aspects of the culture of medicine - from the hierarchical structure to problems related to work-life balance. But despite increasing lip service to these concerns, many workplace challenges remained entrenched.

Reports on the grim conditions of rest spaces for resident doctors provoked gasps at the Canadian Conference on Medical Education. From dirty sheets and bathrooms, to broken lights and poor security, residents warned that the state of some call rooms threatened physician

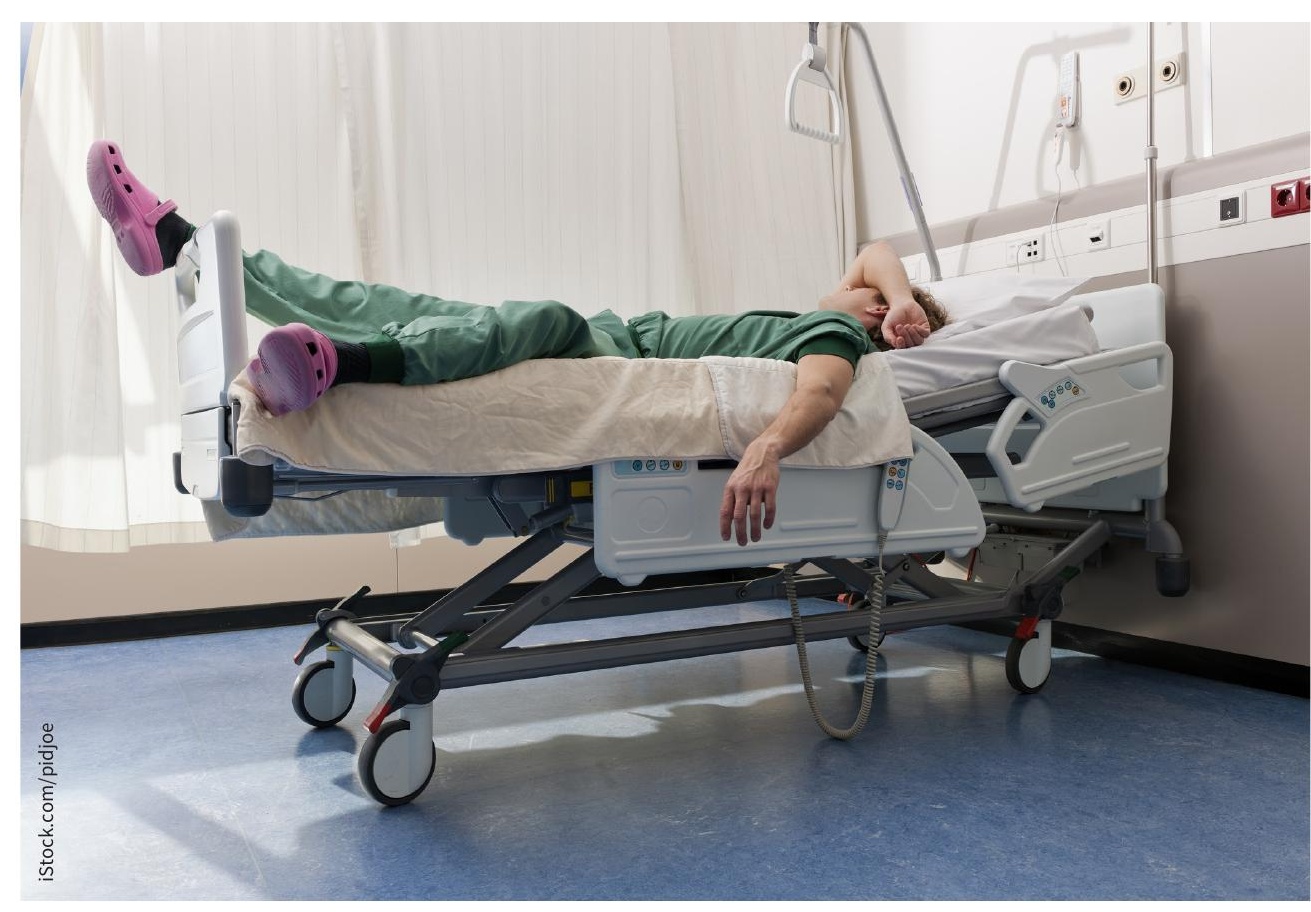

well-being. They proposed minimum national standards, including basics like clean linen and functioning toilets.

Spaces for doctors to unwind and socialize have disappeared over the years due to cost-cutting and hospital overcrowding. But 2019 saw a renewed appreciation for the doctor's lounge, with physicians in the United Kingdom and the United States calling for the revitalization of common spaces to help fight burnout.

Attitudes about taking personal leave appeared to improve with growing awareness of the high rates of burnout among doctors. Still, many trainees were reluctant to ask for time off, even if their contracts allowed it, because it put extra strain on their colleagues.

The decades-long debate over resident duty hours showed no signs of
With greater awareness of burnout in medicine in 2019, focus shifted to the workplace conditions

undermining physician health. 
subsiding. One study found no major differences in patient outcomes in the United States before and after reforms were introduced to limit resident working hours. However, trainee doctors argued that it's still worth limiting duty hours for the sake of their own health.

Some doctors questioned whether long working hours and night shifts contribute to miscarriages among physicians. One survey of physicians found that just over half reported a miscarriage and none were aware of workplace accommodations for early pregnancy. Physicians shared stories of working through miscarriages and not disclosing pregnancies for fear of facing professional reprisal.

\section{Discrimination}

Physicians also shone a spotlight on various forms of discrimination in medicine, including anti-fat bias. According to data presented at the Canadian Obesity Summit, the more people are exposed to weight bias and discrimination, the more likely they are to gain weight and become obese, even if they were thin to begin with. They're also more likely to die from any cause, regardless of body mass index.

When it comes to ageism, training is part of the problem. The Canadian Geriatrics Society found that Canadian medical schools teach less than threequarters of the group's recommended core competencies in the care of older patients.

However, Canadian medical schools made strides to improve the cultural safety and inclusiveness of training. As part of a joint commitment to action on Indigenous health, Canada's 17 medical schools will ensure a minimum number of Indigenous students are admitted each year, build a critical mass of Indigenous faculty, and implement new curricula with a focus on anti-racism and anti-colonialism. The Royal College of Physicians and Surgeons of Canada will also require all programs to include cultural safety training.

\section{Health human resources crisis}

Doctors at the Canadian Medical Association Health Summit warned that Canada's wait times crisis is a health human resources crisis, citing the country's low number of physicians per capita. According to one speaker, compared with growing demand for health services from aging patient populations, the global health workforce is shrinking.

Canadians elected a minority Liberal government on a promise to ensure that everyone has access to a family doctor or primary care team. But some questioned the feasibility of the promise, and the pledge was light on details beyond a commitment to work with the provinces and territories.

Although the total number of doctors in Canada has grown at rates faster than the population for more than a decade, family medicine has struggled to attract physicians to its ranks. Family medicine residency programs continued to report the most unfilled positions in Canada's residency match, accounting for 138 of a total 209 empty seats after the first iteration of the 2019 match.

Some provinces looked to recruit foreign physicians to make up shortfalls in local supply, raising questions about the ethics of poaching physicians from countries like England and Scotland, which are facing shortages of their own.

Others proposed national licensing as a potential solution to doctor shortages. Current licensing rules pose a barrier to doctors working wherever they are most needed and limit the reach of telemedicine programs. The Canadian Medical Association and other national medical organizations launched a task force to explore the regulatory changes needed to allow doctors to deliver telemedicine across provincial and territorial borders.

\section{Cannabis and vaping}

Emergency physicians and public health experts called for tight regulations on cannabis edibles and concentrates, warning that children tend to mistake the products for ordinary treats. More than a dozen children suffered serious or life-threatening events linked to recreational cannabis in the months around legalization, and at least six cases involved edibles that were not legal at the time.

Emergency doctors also warned that cases of a nausea-inducing illness linked to cannabis are underdiagnosed due to a lack of awareness about the condition. One emergency physician in Toronto said that he often sees one patient with cannabinoid hyperemesis syndrome per shift; the illness is marked by repeated and severe bouts of vomiting after prolonged, excessive cannabis use.

Worries about the explosion of popularity of vaping products among Canadian teens proved prescient. In the fall, the US Centers for Disease Control and Prevention reported hundreds of cases of severe pulmonary disease linked to vaping. Cases in Canada followed, with no single product linked to all cases. However, later investigations found that most of the people had used products containing $\mathrm{THC}$, the main psychoactive compound in cannabis.

Lauren Vogel, CMAJ 